\title{
Incidental task sequence learning: perceptual rather than conceptual?
}

\begin{abstract}
In four experiments we investigated whether incidental task sequence learning occurs when no instructional task cues are available (i.e. with univalent stimuli). We manipulated task sequence by presenting three simple binary-choice tasks (colour, form or letter case decisions) in regular repeated or random order. Participants were required to use the same two response keys for each of the tasks. We manipulated response sequence by ordering the stimuli so as to produce either a regular or a random order of left versus right-hand key presses. When sequencing in both, or either, separate stream (i.e. task sequence and/or response sequence) was changed to random, only those participants who had processed both sequences together showed evidence of sequence learning in terms of significant response time disruption (Experiments 1-3). This effect disappeared when the sequences were uncorrelated (Experiment 4). The results indicate that only the correlated integration of task sequence and response sequence produced a reliable incidental learning effect. As this effect depends on the predictable ordering of stimulus categories, it suggests that task sequence learning is perceptual rather than conceptual in nature.
\end{abstract}

\section{Introduction}

Learning is a fundamental human ability which often occurs incidentally, that is, without awareness and without deliberate intention. Following on from

Josephine Cock and Beat Meier have contributed equally to this paper. We simply tossed a coin to determine the order of authorship.

J. Cock · B. Meier $(\bowtie)$

Department of Psychology, University of Bern, Muesmattstr. 45, 3000 Bern 9, Switzerland

E-mail: beat.meier@psy.unibe.ch

Tel.: +41-31-6314039

Fax: + 41-31-6318212
Lashley's (1951) pioneer work on the problem of serial order in behaviour, and Nissen and Bullemer's $(1987)^{1}$ famous serial reaction time (SRT) task, there is ample evidence that fairly complex structures, such as sequences of perceptual stimuli and sequences of motor responses, can be learned incidentally (Cock et al. 2002; Koch and Hoffmann 2000; Marsolek and Field 1999; Perruchet and Amorim 1992; Shanks and Johnstone 1999; Willingham et al. 1993, amongst many others). In the present study, we investigate whether an even more sophisticated kind of sequence can be learned unintentionally, in particular whether incidental sequence learning extends to sequences of tasks. This, of course, brings into question the mechanism underlying sequence learning (Goschke 1998; Shanks and Johnstone 1998; Willingham 1998). For example, is it stimulus-based, response-based or both (Hoffmann and Sebald 1996; Nattkempfer and Prinz 1993; Ziessler 1994)? Is it a kind of motor or visuo-spatial learning, or both at once (Bapi et al. 2000; Cohen et al. 1990; French and Miner 1995; Koch and Hoffmann 2000; Mayr 1996)? Can it involve concepts as well as percepts?

So far, incidental task sequence learning has been addressed by three previous studies (Heuer et al. 2001; Gotler et al. 2003; Koch 2001). These studies have in common that ambivalent stimuli were used, that is, stimuli with features relevant to more than one task. As a consequence, it was necessary for the experimenters to present instructional cues to indicate which task needed to be performed on each trial. While all of these studies

\footnotetext{
${ }^{1}$ In the typical SRT (e.g. Nissen and Bullemer 1987), a single visual stimulus appears at one of several possible locations and participants make a key-press response. On subsequent trials, the stimulus is presented at different locations either according to a repeating sequence or at random. With training, reaction times (RTs) decrease, but when randomly ordered stimuli are inserted, RTs increase (decreasing again when the sequence is reinstated). These findings are taken to indicate sequence learning. Unlike Nissen and Bullemer's incidentally trained participants, the majority of whom reported awareness of the sequence, participants in other studies showed changes in RTs in the absence of explicit knowledge (e.g. Willingham et al. 1989, 1993).
} 
found evidence of incidental learning of some kind, effects were attributed to different sources.

Koch (2001) used a sequence of simple binary-choice tasks, in which participants identified the colour, the form, or the size of just one kind of stimulus (i.e. ambivalent stimuli). Before the presentation of each stimulus, an external cue (dollar sign, arrow, or yellow square) indicated which of the three tasks was to be performed. Whether the task required a left or righthand key response was random. Most importantly, sequence learning effects (measured in terms of differences in response times between sequenced and random blocks) were found, and they were greater when the response-cue interval was short (100 ms vs $900 \mathrm{~ms})$, but non-existent when the cue-stimulus interval was long (900 ms vs $100 \mathrm{~ms}$ ). Koch concluded that learning of the cue types led to pre-activation of the task sets. Inevitably, however, the instructional cues also followed a sequence. Therefore, learning effects might have been perceptually rather than conceptually based.

Using pairs of digits as stimuli, Heuer et al. (2001) presented a sequence of four dimensionally organized tasks (digit magnitude vs digit location judgements and compatible vs incompatible judgement-to-response mappings). Vertical location of the digits indicated the kind of judgement required and stimulus colour indicated the type of mapping required (Experiment 1 and 2). Apart from initial instructions to participants, there were no other, i.e. external, trial-by-trial task cues. Heuer et al. found evidence of incidental sequence learning, but suggested that it was restricted to the learning of a sequence of "uninterpreted" stimulus features rather than the task sequence itself. That is, learning the order of the perceptual cues was probably sufficient to account for the results without reference to pre-activation of conceptual task sets.

Gotler et al. (2003) conducted a study in which two different types of instructional task cues were used (horizontally vs vertically aligned arrows and horizontal vs vertical lines). In one condition, cue type was held constant, and in the other, it varied at random. The results showed no difference in sequence learning between conditions. Gotler et al. argued that this is a strong indication of proper task sequence learning. However, the two types of instructional cue used by Gotler et al. were perceptually rather similar. Therefore, an alternative interpretation is that learning of a perceptually based sequence of cues occurred and this alone was responsible for the results.

According to this line of thought, similarities between the instructional cues were sufficient for participants in the study by Gotler et al. to form generalized representations of them. Such generalizations would be akin to the formation of perceptually based categories, for example, "horizontal markings" and "vertical markings". Studies on early vision and visual search have shown that perceptually based, data-driven processing of the physical features of stimuli are rather sophisticated and can result in the unitization of different kinds of stimuli (Ahissar and Hochstein 1995; Goldstone 2000; Humphreys et al. 1989; Krueger 1984; Wolfe et al. 1992). More importantly, in Gotler et al.'s study, the generalized groupings of task-cues would have formed a perceptually based sequence that correlated directly with the task sequence. As proper incidental task sequence learning would imply the processing of sequential components of a conceptual rather than perceptual kind, we feel further investigation is warranted. By conceptual we mean a more cognitive kind of mental representation.

In the present study, we propose a different approach for investigating the nature of incidental task sequence learning. In contrast to the previous studies, we used univalent stimuli. Stimuli were unique to each specific task and did not share any relevant features with stimuli of the other tasks (cf. Woodward et al. 2003). With this restriction, no instructional task cues were necessary to indicate which task was to be performed on any trial. To investigate whether task sequence learning is perceptually or conceptually based, we exposed participants to a task sequence as well as an independent motor response sequence. The presence or absence of the task sequence and the presence or absence of the response sequence were manipulated orthogonally.

We reasoned that if task sequence learning occurs in a condition where tasks but not responses are sequenced, this would suggest proper (i.e. conceptually based) task sequence learning. However, if task sequence learning occurs only in a conjoint condition, where tasks and responses are sequenced in unison (i.e. correlated sequences), this would suggest perceptual rather than conceptual learning effects. Only in this condition, at least in our experiments, does a predictable series of stimulus categories emerge. By stimulus categories, we refer to those generalized groupings of stimuli which, in accordance with the various different tasks (described below), required a common response (e.g. angular shapes, red figures, etc.). It should be noted here that, in conditions where it occurred, participants' attention was not drawn to the existence of these "common response" groupings. Rather, in all conditions, instructions to participants were focussed on performance of the tasks themselves and the need to switch between them, for example, having to decide if a shape was rounded or angular, followed by having to decide if a letter was upper- or lowercase, etc.

In all, we report four experiments in which switching between three tasks was required, namely between colour decisions for coloured fuzzy figures (blue vs red), shape decisions for geometric shapes (rounded vs angular) and case decisions for letters (upper- vs lowercase). In order to disguise the task sequence, we required the participants to use just two response keys, that is, the same two keys for each of the three binarychoice tasks. As a consequence our arrangement involved using only left versus right-hand responses, compared to four or six keys mapped to separate fingers in most other SRT task studies. Also, each of the two response keys (i.e. left vs right) corresponded to three 
different types of stimuli (i.e. blue, rounded, uppercase vs red, angular, lowercase) while normally in SRT tasks there is an isomorphic relation between keypad arrangement and spatial locations of stimuli on screen (Nissen and Bullemer 1987).

All experiments consisted of eight blocks, two random practice blocks and six experimental blocks. The critical comparison was between experimental blocks 6 and 8 and pseudo-random block 7. By "pseudo-random" we refer to an ordering that was statistically comparable to the sequenced ordering in terms of frequency of components and transitions but without the regular predictability of the transitions. We deliberately chose to use a pseudo-random block rather than introduce a new sequence so that we could compare experimental conditions to a completely pseudo-random control group throughout the eight blocks. By "completely pseudo-random control group", we refer to the condition in which neither the task order nor the response order was sequenced at any time.

In Experiments 1-3, the task sequence and the response sequence were of the same length and were thus correlated. In Experiment 1, 12-element sequences were used for both tasks and responses, and in Experiments 2 and 3, 6-element sequences were used. In Experiment 1 and 2, both the task sequence and the response sequence were changed to pseudo-random at block 7. In Experiment 3, either the task sequence or the response sequence was changed to pseudo-random at block 7 . Finally, in Experiment 4, the length of the sequences was altered such that a 6-element sequence was used for tasks and an 8-element sequence for responses. Therefore, the sequences were uncorrelated ${ }^{2}$ (cf. Schmidtke and Heuer 1997; Shin and Ivry 2002).

To anticipate the main results, we found evidence of sequence learning in Experiments 1 and 2, but only when task order and response order were sequenced at once. Experiment 3 revealed comparable disruption when just one sequence at a time rather than both together, was changed to random. In Experiment 4, no learning effect was found using uncorrelated sequences.

\section{General method}

\section{Materials}

Stimuli were approximately $4 \times 3 \mathrm{~cm}$ in size, and shown against a pale grey background. For the letter task (upper vs lowercase), six different letters were used, for the shape task (rounded vs angular) six different geometric shapes and for the colour task (red vs blue) six different fuzzy figures. An example of each type is shown in Fig. 1. Stimuli were presented centrally, on a 15-in. high-resolution colour monitor, connected to an IBM-

\footnotetext{
${ }^{2}$ In the short term, the different length sequences were uncorrelated Their combination was, however, repeated every 24 trials.
}

compatible personal computer and an external response pad. Viewing distance was approximately $50 \mathrm{~cm}$.

Across experiments, four kinds of experimental conditions were used, resulting from the orthogonal combination of task order (sequenced vs random) and response order (sequenced vs random). In one condition (Experiments 1-4), a sequenced order of three simple decision tasks, combined with a separately sequenced order of required responses, was presented (we will refer to this group as the "seq-seq" condition). In a second condition, tasks were sequenced, but responses were ordered at random (i.e. the "seq-ran" condition). In a third condition, tasks were ordered at random, but responses were sequenced (i.e. the "ran-seq" condition), and in the fourth condition, the control condition, both streams were ordered at random throughout (i.e. the "ran-ran" condition). The seq-ran, ran-seq and ran-ran conditions were administered in Experiments 1 and 2 only.

For the seq-seq and seq-ran conditions, task order was sequenced according to a repeating cycle. Two different sequenced orders were used for counterbalancing within conditions. For the seq-ran and ran-ran conditions, a statistically comparable order of tasks was created pseudo-randomly with the same frequency characteristics but unpredictable transitions. Neither the sequenced nor the random order featured sequential task repetitions and each task occurred equally often in each block.

For the seq-seq and ran-seq conditions, response order was sequenced according to a repeating cycle. For the ran-seq and ran-ran conditions, a statistically comparable order of responses was created pseudo-randomly excluding long runs of either left or right responses and ensuring that the same overall number of left and right responses, and comparable numbers of switch and repeat transitions, had to be made. For all conditions, whilst complying with the task sequence and/or response sequence, stimulus presentation varied at random.

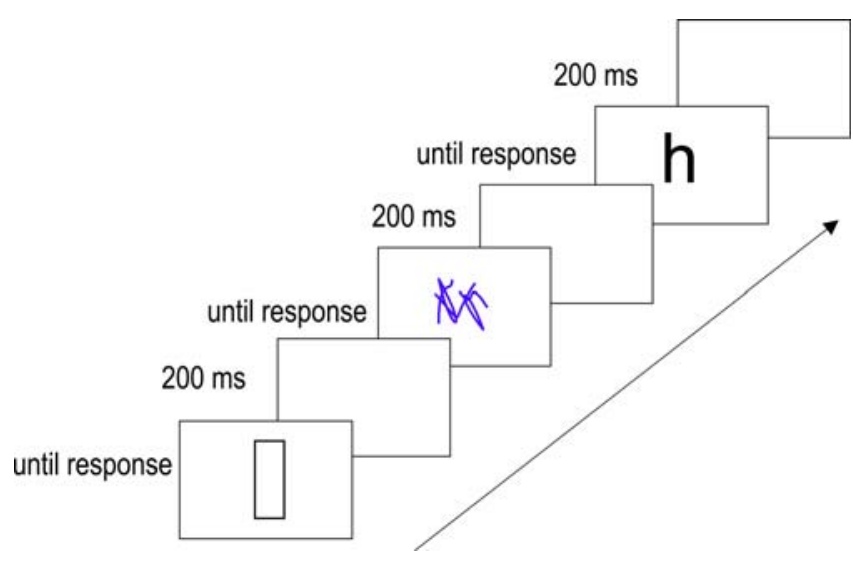

Fig. 1 Example of procedure and stimuli 


\section{Procedure}

Participants were tested individually, with instructions given verbally and on screen. They were told that the experiment concerned effects of practice on speed of performance of simple tasks. They were informed that the experiment comprised three separate tasks. On some trials they would be required to identify the colour of meaningless fuzzy figures (red or blue?), on other trials the form of geometric shapes (rounded or angular?), and on yet other trials the format of letters (lowercase or uppercase?). It was explained that each stimulus would remain on screen until the participant pressed either response key (left vs right hand, index fingers). Two initial practice blocks (one comprising 48 random trials and one comprising 96 random trials) trained participants on the appropriate stimulus to response key mappings. Participants were instructed to respond as quickly and as accurately as possible. They were told that if they made mistakes, they should simply continue. When the participant was ready, the experimenter pressed a key to initiate the blocks of trials. Each stimulus remained on screen until the participant pressed one of the two response keys, followed by an interval of $200 \mathrm{~ms}$ before presentation of the next stimulus (see Fig. 1). The two initial practice blocks were followed by four experimental blocks (blocks 3-6, each comprising 96 trials) and one pseudo-random block (block 7, 96 trials). ${ }^{3}$ In block 8 , the original sequenced (or pseudo-randomized) ordering was reinstated. There was a brief pause between blocks. No feedback on performance was provided.

After the test session, which lasted approximately 20 min, participants were specifically asked if they had noticed anything particular about the ordering of the stimuli. This gave them the opportunity to report, of their own accord, the task sequence, the response sequence and/or the possible integration of the two. All participants were subsequently told that two repeating sequences had been present during most of the blocks, one involving the order of the three tasks, and the other involving the order of the correct response key presses. They were asked to generate these sequences, once each and in whatever order they preferred, in abbreviated words, either from memory or by guessing and to give as many element transitions as they thought appropriate. No feedback was provided.

\section{Data analysis}

For all experiments, trials on which errors that were made were excluded from analysis. Average error rate was less than $5 \%$ throughout and comparable between

\footnotetext{
${ }^{3}$ We used the same random ordering in block 7 for all conditions rather than a counterbalanced ordering of different kinds of sequences in order to provide a direct comparison with the ran-ran conditions. Furthermore, introducing an entirely new sequence at block 7 might have led to other (i.e. unwanted) correlations and similarities between sequences.
}

conditions and experiments. Reaction time data for the three decision tasks were aggregated and median RTs per block were computed for each individual participant. For purposes of analysis and interpretation, decreasing RTs over blocks 3-6 were taken as directly indicative of a general training effect (also possibly including some sequence learning). Increased RTs at block 7 (where sequences were simultaneously changed to pseudo-random) were taken as indirectly indicative of sequence learning. Disruption scores were calculated as the difference between performance in block 7 and the average of blocks 6 and 8. For all statistical analyses, an alpha level of .05 was used. Degrees of freedom and $M S E$ values were Greenhouse-Geisser adjusted when appropriate.

\section{Participants}

The number of participants per experimental condition was 20 and was kept constant across experiments (Table 1). All were undergraduate students from the University of Bern who took part in return for course credit. None of the students participated in more than one experiment.

\section{Experiment 1}

The aim of Experiment 1 was to establish first whether task sequence learning occurs when tasks are performed using univalent stimuli, that is, under conditions where no instructional cue is necessary to indicate which task is to be performed, and second whether some form of repeating, perceptually based representation is necessary for task sequence effects to be found. By perceptually based representation, we specifically refer to the additional existence of a sequence of stimulus categories (such as red figures, small letters, etc.) made possible by the correlation of a sequence of binary-choice, conceptually based tasks (such as colour decisions, letter-case decisions, etc.) with a concurrent sequence of required motor responses (such as left vs right hand key presses).

\section{Method}

\section{Design}

Participants were randomly assigned to one of four experimental conditions: seq-seq, seq-ran, ran-seq and ran-ran. Task order and response order were manipulated between subjects, whilst block was manipulated within subjects, thus resulting in a mixed design.

\section{Materials}

For the seq-seq and seq-ran conditions, task order was sequenced according to one of two (counterbalanced) 
Table 1 Number of participants with relevant explicit knowledge, together with mean disruption scores (MDS) measured in ms

\begin{tabular}{|c|c|c|c|c|c|c|c|c|c|c|}
\hline \multirow{2}{*}{$\begin{array}{l}\text { Experiment } \\
\text { Condition }\end{array}$} & \multicolumn{2}{|c|}{ Neither sequence } & \multicolumn{2}{|c|}{$\begin{array}{l}\text { Task } \\
\text { only }\end{array}$} & \multicolumn{2}{|c|}{$\begin{array}{l}\text { Response } \\
\text { quence only }\end{array}$} & \multicolumn{2}{|c|}{ Both sequences } & \multicolumn{2}{|c|}{ All participants } \\
\hline & $n$ & MDS (SD) & $N$ & MDS (SD) & $n$ & MDS (SD) & $N$ & MDS (SD) & $n$ & MDS (SD) \\
\hline \multicolumn{11}{|l|}{ Experiment 1} \\
\hline seq-seq & 19 & $45(44)$ & 0 & - & 0 & - & 1 & 135 & 20 & $50(48)$ \\
\hline seq-ran & 20 & $-8(31)$ & 0 & - & 0 & - & 0 & - & 20 & $-8(31)$ \\
\hline ran-seq & 19 & $4(27)$ & 0 & - & 1 & -19 & 0 & - & 20 & $3(27)$ \\
\hline ran-ran & 20 & $2(20)$ & 0 & - & 0 & - & 0 & - & 20 & $2(20)$ \\
\hline \multicolumn{11}{|l|}{ Experiment 2} \\
\hline seq-seq & 3 & $57(39)$ & 0 & - & 12 & $118(125)$ & 5 & $33(28)$ & 20 & $87(105)$ \\
\hline seq-ran & 16 & $-8(43)$ & 0 & - & 4 & $-23(80)$ & 0 & - & 20 & $-11(50)$ \\
\hline ran-seq & 12 & $-7(55)$ & 0 & - & 8 & $11(41)$ & 0 & - & 20 & $0(49)$ \\
\hline ran-ran & 15 & $2(30)$ & 0 & - & 5 & $21(18)$ & 0 & - & 20 & $7(28)$ \\
\hline \multicolumn{11}{|l|}{ Experiment 3} \\
\hline seq-seq (b7 ran-seq) & 4 & $8(56)$ & 0 & - & 14 & $65(79)$ & 2 & $20(28)$ & 20 & $49(74)$ \\
\hline seq-seq (b7 seq-ran) & 5 & $66(20)$ & 0 & - & 14 & $55(52)$ & 1 & 109 & 20 & $60(31)$ \\
\hline \multicolumn{11}{|l|}{ Experiment 4} \\
\hline seq-seq (uncorrelated) & 13 & $-7(58)$ & 0 & - & 7 & $21(44)$ & 0 & - & 20 & $3(54)$ \\
\hline
\end{tabular}

Note: Individual disruption scores are calculated according to the difference in median RTs in block 7 compared to median RTs for the average of blocks 6 and 8

repeating 12-element cycles (i.e. letter-task, colour-task, shape-task, colour-task, shape-task, letter-task, shapetask, colour-task, letter-task, colour-task, letter-task, shape-task and shape-task, colour-task, shape-task, letter-task, colour-task, letter-task, shape-task, colour-task, letter-task, shape-task, letter-task, colour-task for counterbalancing within condition). For the ran-seq and ranran conditions, task order was pseudo-random such that across each block the three tasks were equally distributed and without successive task repeats. Statistically, that is, in terms of number of transitions between each of the six possible task orders, random ordering was comparable to sequenced ordering. For the seq-seq and ran-seq conditions, response order was sequenced according to a repeating 12 -element cycle, (i.e. $R-L-R-R$ $L-R-R-R-L-L-L-L$, or the opposite for counterbalancing within condition). For the seq-ran and ran-ran conditions, response order was pseudo-random. Across each block (randomized separately), this ordering comprised equal numbers of $\mathrm{L}$ and $\mathrm{R}$ key presses and no runs of more than 4 same-side responses. Switch and repeat transitions occurred at roughly the same rate as those in the sequenced response ordering. The task sequence and the response sequence were obviously different in structure, the former having three components and no repetitions, the latter having two components and several repetitions. However, our aim was not to compare different forms of sequence structure. We simply used this arrangement in order to create the integrated condition (seq-seq) without making the correlation obvious.

\section{Results}

The results of Experiment 1 are shown in Fig. 2. RTs decreased initially for all groups. The mean decrease from block 3 to block 6 was $65 \mathrm{~ms}(\mathrm{SD}=105)$ for the seq- seq condition, $58 \mathrm{~ms}(\mathrm{SD}=87)$ for the seq-ran condition, $75 \mathrm{~ms}(\mathrm{SD}=99)$ for the ran-seq condition and $32 \mathrm{~ms}$ $(\mathrm{SD}=72)$ for the ran-ran condition. Inspection of Blocks 6-8 indicates that only participants in the condition with both task order and response order sequenced (seq-seq) appear to be disrupted by the introduction of pseudorandom block 7 . The corresponding mean disruption scores are presented in Table 1.

Statistical analyses were conducted separately for blocks 3-6, and for blocks 6-8. A repeated measures analysis of variance (ANOVA) for blocks 3-6 revealed a significant main effect of block, $F(1.71,130.03)=31.64$, $\mathrm{MSE}=2644.17, P<0.001$. No other main effect and no interaction reached significance (all $F_{\mathrm{s}} \leq 2.53$ ).

To assess the effect of the pseudo-random block 7, a separate $2 \times 2 \times 2$ ANOVA was conducted, comparing performance on block 7 to the average of performance on blocks 6 and 8 , and again, comparing sequenced versus pseudo-random ordering with respect to both tasks and required responses. This enabled the separation of specific task or response effects (i.e. sequence learning), from more general training effects. Three significant interactions emerged, namely block $\times$ task order, with $F(1,76)=6.38, P<0.02$, block $\times$ response order, $F(1,76)=15.99, P<0.001$, and block $\times$ task order $\times$ response order, $F(1,76)=14.94, P<0.001$ (all MSE $=546.96$ ). A one-way ANOVA of the disruption scores revealed a significant effect, with $F(3,76)=12.43$, $P<0.001$. Post-hoc Tukey HSD tests showed that the significant differences between groups pertained to the seq-seq condition compared to each of the three other conditions (all $P \mathrm{~s}<0.05$ ).

A summary of the results of the explicit knowledge test is presented in Table 1. Only two participants showed substantial explicit knowledge. One of these (seq-seq) correctly reported more than half of both sequences (disruption score $=135 \mathrm{~ms}$ ), and spontane- 
Fig. 2 Group mean RTs for Experiment 1. seq-seq: task order and response order both sequenced. seq-ran: task order sequenced, response order randomized. ran-seq: task order randomized, response order sequenced. ran-ran: task order and response order both randomized. For all conditions, blocks 1,2 and 7 comprised separately randomized task and response orders

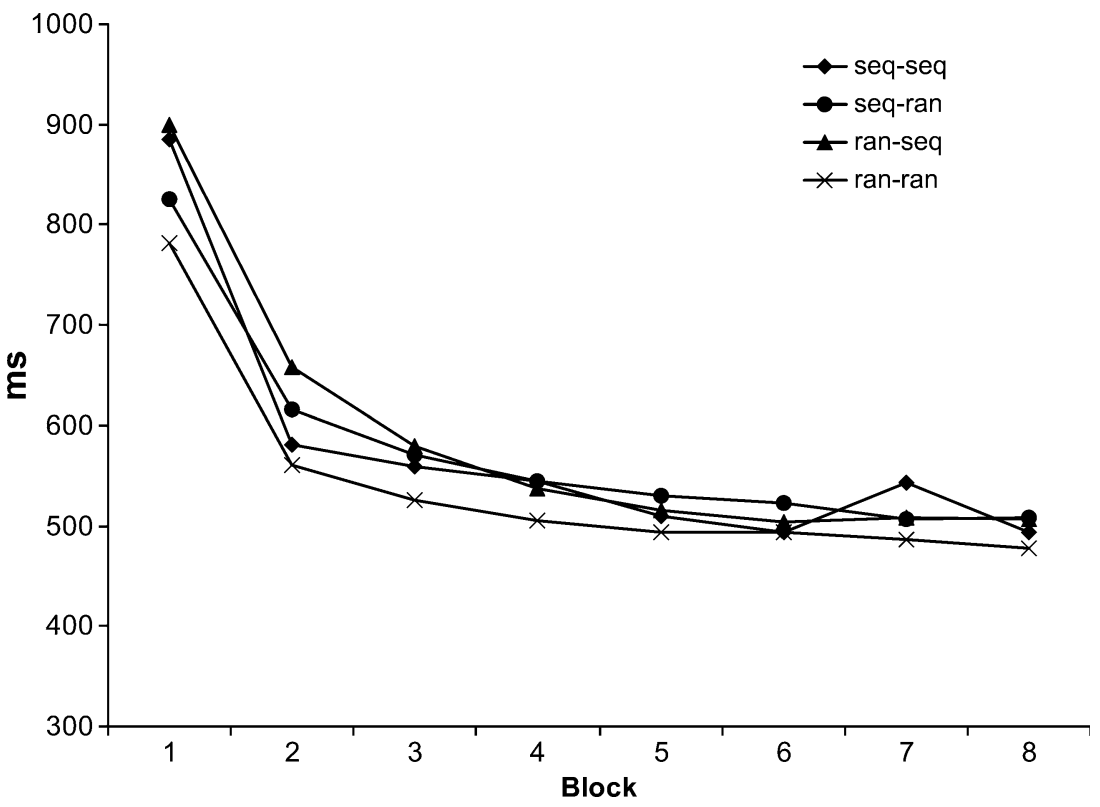

ously reported four elements of the inherent stimulus category sequence. The other participant (ran-seq) correctly reported more than half of the response sequence and, not surprisingly, none of the task sequence other than chance level suggestion (disruption score $=-19 \mathrm{~ms}$ ). The majority of participants found it difficult to generate information.

\section{Discussion}

The purpose of Experiment 1 was to test whether task sequence learning can be found when switching between tasks is performed in the absence of instructional cues and when the stimuli are univalent. The data, however, gave little indication that either the task sequence or the response sequence was learned when sequences were manipulated separately. Two experimental conditions (seq-ran and ran-seq) were statistically indistinguishable from the pseudo-random control (ran-ran). However, the conjoint condition (seq-seq), in which tasks and responses were sequenced in parallel, showed an incidental learning effect in terms of significant disruption of RTs at block 7. One participant who had explicit knowledge of both sequences showed considerable disruption (disruption score $=135 \mathrm{~ms}$ ). However, the other 19 participants in the same condition, who had no explicit knowledge, were also disrupted (mean disruption scor$\mathrm{e}=45 \mathrm{~ms}$ ).

This finding seems to relate to the fact that the sequences (seq-seq) could be successfully integrated (Schmidtke and Heuer 1997). Here, the existence of a sequence of generalized stimulus categories was made possible by the correlation of the task sequence and the required response sequence. While the task sequence was essentially conceptual and the response sequence was essentially motor, the resultant stimulus category sequence was, very likely, perceptual. This was only possible in the correlated seq-seq condition.

For the separately sequenced conditions (seq-ran and ran-seq), it seems likely that the 12-element sequences were simply too long to be learned incidentally when presented in isolation. That is, as the sequences comprised only a small number of components (i.e. three different tasks, two different responses), parsing them into smaller chunks would have reduced element transitions to chance level combinations. Therefore, we conducted a second experiment, in which the length of the sequences was reduced. With this manipulation, we expected to enhance the chance of finding sequence learning in conditions where tasks and responses were sequenced separately.

\section{Experiment 2}

The aim of Experiment 2 was to replicate the sequence learning effect found in Experiment 1, and to investigate task sequence learning further by means of using a shorter sequence length. Although 12-element sequences have mostly been the norm in stimulus-response sequence learning since Nissen and Bullemer's seminal study (1987), shorter sequences have been preferred in task sequence learning. Koch, for example, used a nineelement sequence and Gotler et al. and Heuer et al. used eight-element sequences. By using a six-element sequence, we expected to enhance the chance of finding task sequence learning. Although the sequence length was half that of Experiment 1, the total number of trials per block remained unchanged. Consequently, participants in Experiment 2 processed the sequences more often. 
Method

Design

Conditions and design were identical to those in Experiment 1.

\section{Materials and procedure}

These were exactly as in Experiment 1, the only exception being sequence length and number of cycles per block. For the seq-seq and seq-ran conditions, task order was sequenced according to one of two repeating sixelement cycles (i.e. colour-task, shape-task, letter-task, shape-task, colour-task, letter-task and letter-task, colour-task, shape-task, colour-task, letter-task, shape-task for counterbalancing within condition). For the ran-seq and ran-ran conditions, task order was pseudo-random. Across each block, the three tasks were equally and evenly distributed comparable to the sequenced task order and with no task repeats. For the seq-seq and ranseq conditions, response order was sequenced according to a repeating six-element cycle, (i.e. $L-R-L-L-R-R$ or the opposite for counterbalancing within condition). For the seq-ran and ran-ran conditions, response ordering was pseudo-random. Across each block, this comprised equal numbers of $\mathrm{L}$ and $\mathrm{R}$ key presses and no runs of more than three same-side responses. Switch and repeat transitions occurred at roughly the same rate as those in the sequenced response ordering.

Results

The results of Experiment 2 are shown in Fig. 3. As expected, RTs decreased initially for all groups. Overall, the data pattern looks very similar to that of Experiment 1. However, it seems that two groups (seq-ran and ran$s e q$ ) were generally slower than the control (ran-ran). As this difference was evident from block 1 onwards, it appears to be a chance effect of assignment to conditions. The other group (seq-seq) shows comparable performance to the control group in the practice blocks 1 and 2, and then diverges slightly across blocks 3-6, suggestive of a steeper training/learning curve. Mean decrease from block 3 to block 6 was $109 \mathrm{~ms}(\mathrm{SD}=132)$ for the seq-seq condition, $71 \mathrm{~ms}(\mathrm{SD}=124)$ for the seqran condition, $66 \mathrm{~ms}(\mathrm{SD}=84)$ for the ran-seq condition, and $40 \mathrm{~ms}(\mathrm{SD}=74)$ for the ran-ran condition. Also similar to Experiment 1, only participants in the seq-seq condition seem to be disrupted by the introduction of pseudo-random block 7 .

A repeated measures ANOVA across blocks 3-6 confirmed a significant main effect of block, $F$ (1.91, $145.05)=25.45, \mathrm{MSE}=4619.89, P<0.001$, and a significant task order $\times$ response order interaction, $F(1$, 76) $=5.64, \mathrm{MSE}=129270.67, P=0.02$. There was no main effect of either task order or response order (both $F<1)$. None of the interactions with block reached significance (all $F_{\mathrm{s}}<2$ ), suggesting that the slope of the learning effect was statistically comparable across the four conditions.

A separate $2 \times 2 \times 2$ ANOVA was conducted, comparing performance on block 7 to the average of blocks 6 and 8 , and again, sequenced versus pseudo-random ordering with respect to both tasks and required responses. A significant main effect of block (i.e. increased RTs at block 7) was found, $F(1,76)=8.34$, MSE $=2087.58, P<0.01$, and the task order $\times$ response order interaction was significant, $F(1,76)=4.21$, $\mathrm{MSE}=45028.11, P<0.05$. More importantly, as in Experiment 1 , three significant interactions with block emerged, namely block $\times$ task order $F(1,76)=5.70$,
Fig. 3 Group mean RTs for Experiments 2 and 3. seq-seq: both task order and response order sequenced. seq-ran: task order sequenced, response order randomized. ran-seq: task order randomized, response order sequenced. ran-ran: task order and response order both randomized. For all conditions, blocks 1 and 2 comprised separately randomized task and response orders. In Experiment 2, block 7 also comprised separately randomized task and response orders. In Experiment 3 , block 7 comprised either continuation of the sequenced task order together with randomized response order (block 7 seq-ran) or randomized task order together with continuation of the sequenced response order (block 7 ran-seq)

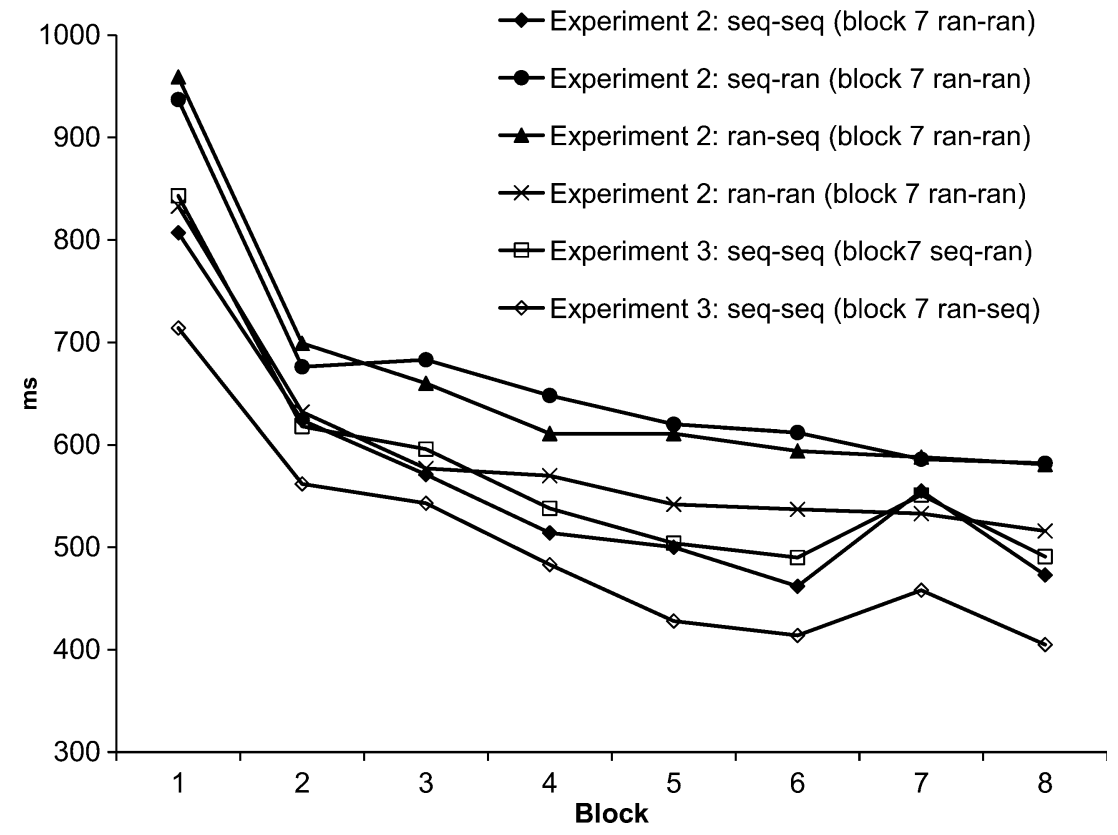


$P<0.02$, block $\times$ response order, $F(1,76)=10.14$, $P<0.01$, and block $\times$ task order $\times$ response order, $F(1$, $76)=13.21, P=0.001$, all $\mathrm{MSE}=2087.58$. These interactions are qualified by the fact that the increase at block 7 pertains predominantly to the seq-seq condition. A one-way ANOVA confirmed a difference between conditions, with $F(3,76)=9.69, P<0.001$. Post-hoc Tukey HSD tests showed that the significant differences between groups pertained only to the seq-seq condition compared to each of the three other conditions (all $P$ s $<0.05)$.

A summary of the results of the explicit knowledge test is presented in Table 1. Five seq-seq participants (mean disruption score $=33 \mathrm{~ms}$ ) correctly generated more than half of the response sequence (one generated all six transitions) as well as more than half of the task sequence (two generated all six transitions). They also spontaneously generated most or all of the inherent stimulus category sequences. Another $12 \mathrm{seq}$-seq participants correctly generated more than half of the response sequence but little (if anything) of either the task sequence or the stimulus category sequence. These 12 participants were largely, but not exclusively, responsible for the mean disruption score at block 7 (118 ms). Three other seq-seq participants showed disruption $(57 \mathrm{~ms})$ despite an absence of relevant explicit knowledge.

\section{Discussion}

The results of Experiment 2 replicate the findings of Experiment 1. Only the conjoint sequences condition (seq-seq) showed evidence of sequence learning in terms of disruption when both sequences were simultaneously changed to random. More importantly, this seems to have been related to potentially explicit knowledge of the response sequence rather than the task sequence. We speculate that while it may be possible to notice and remember the response sequence (perhaps by hypothesis testing) in the absence of the existence of the task-sequence, awareness of the actual task sequence may depend on first noticing the sequence of stimulus categories (see below). This, of course, is only possible in the seqseq condition.

The second aim of Experiment 2 was to increase the chance of finding separate sequence learning (tasks or responses) by using shorter sequences. While in the ranseq condition, where correct responses were sequenced but tasks were not, there was some explicit knowledge of the response sequence ( 8 out of 20 participants), this was not generally accompanied by RT disruption at block 7 . We can only speculate that knowledge of the response sequence was acquired after block 7 in the ran-seq condition, or that, in some cases at least, explicit knowledge did not have an influence on speed of performance. As response times were generally fairly fast in all conditions, participants may not have found the experiment very onerous. It follows that even if some participants (i.e. seq-seq and ran-seq conditions) acquired explicit knowledge of the response sequence early on in the experiment, they may have taken the later disappearance (and reappearance) of the sequence in their stride, so to speak. It is usually assumed in incidental learning studies that performance reflects competence but this need not be so (Kleinsorge et al. 2003). Yet another possibility is that some experimental participants correctly generated sequence parts purely by guessing, as did some of the control participants (see Table 1, seq-ran and ran-ran conditions, response sequence generation).

By design, in the seq-seq condition, the correlated combination of task order and response order resulted in a sequence of stimulus categories. We would argue that participants became sensitive to at least part of this order (for example, red-angular-lowercase-rounded-blueuppercase) because it comprised unique transitions. More importantly, the learning of such a sequence is probably based on perceptual rather than conceptual representations (cf. Goldstone 2000). If so, then the observed disruption in block 7 in Experiments 1 and 2 cannot be attributed to an abstract task sequence learning mechanism. Nor can it be attributed to purely motor-driven sequence learning-otherwise we would have found separate response sequence learning in the ran-seq condition. Only in the seq-seq condition was there a consistent relationship between stimulus categories and required responses (see Hoffmann and Koch 1997; Koch and Hoffmann 2000 for related discussions on the importance of relational structure in sequence learning).

If this line of argument holds, then the introduction of separate randomization at block 7 of either task order or response order should be sufficient to produce the same level of disruption as when both sequences are randomized at once, because in both cases it is the sequence of stimulus categories that disappears. This hypothesis was tested in Experiment 3.

\section{Experiment 3}

In Experiment 3 a slight modification of the seq-seq condition of Experiment 2 was used. In fact, participants in Experiment 3 can be seen as providing two experimental groups additional to Experiment 2. As such, we were able to compare the data from these new conditions directly to the seq-seq condition in Experiment 2. The two new conditions differed from the seq-seq condition only in that the change of the sequence order to pseudorandom at block 7 was implemented separately for tasks and responses. We hypothesized that if the two new groups differed in their disruption scores, this would suggest differential learning of the task sequence and the response sequence. If, however, either or both of the two groups showed less disruption than the conjoint sequences condition of Experiment 2 (seq-seq), then an additive effect would be implicated. Finally, if all three groups showed comparable disruption, then it would 
seem very likely that the learning of a sequence of stimulus categories had indeed been involved, such that removal of either or both sequences effectively and equally disrupted performance in block 7 .

\section{Method}

\section{Design}

We used a mixed two-factorial design with groups (i.e. type of random ordering in block 7) manipulated between subjects, and blocks manipulated within subjects.

\section{Materials and procedure}

These were exactly as in the seq-seq condition of Experiment 2, with one exception. At block 7, instead of both sequences being simultaneously changed to pseudo-random, for one group only the task sequence changed, and for the other group, only the response sequence changed. These pseudo-random sequences were identical to those used in the ran-seq condition and in the seq-ran condition, respectively, in Experiment 2.

\section{Results}

The results of Experiment 3 are shown in Fig. 3. Overall, the data pattern looks very similar to that of the seq-seq condition of Experiment 2. The mean decrease between block 3 and block 6 for the two new seq-seq conditions was $106 \mathrm{~ms}(\mathrm{SD}=123)$ and $135 \mathrm{~ms}(\mathrm{SD}=124)$, respectively, compared to $109 \mathrm{~ms}(\mathrm{SD}=132)$ for the seq-seq condition in Experiment 2

A repeated measures ANOVA across blocks 3-6 was carried out including all three seq-seq conditions. As expected, a main effect of blocks $F(1.81,103.27)=37.31$, $P<0.001, \mathrm{MSE}=6862.27$ was found, but no other significant effect emerged (all $F \mathrm{~s}<1$ ) The critical comparison concerned the difference between the means of blocks 6 and 8 and block 7. A separate ANOVA revealed a main effect of block with $F(1,57)=44.65$, $\mathrm{MSE}=2895.18, P<0.001$, but no difference between groups $(F(2,57)=2.04, \mathrm{MSE}=40767.03, P>0.1)$. More importantly, there was no block $\times$ groups interaction, $F$ $(2,57)=1.33, \mathrm{MSE}=2895.18, P>0.1$, indicating that all three groups were equally disrupted.

A summary of the explicit knowledge test is presented in Table 1. In the $b 7$ seq-ran condition, one participant (disruption score $109 \mathrm{~ms}$ ) correctly generated more than half of the task sequence, more than half of the response sequence and most of the stimulus category sequence. In the $b 7$ ran-seq condition, two participants (mean disruption score $20 \mathrm{~ms}$ ) correctly generated more than half of all three sequences. In each condition, another 14 participants generated none (or very little) of the task sequence but all or most of the response sequence. They also showed disruption at block 7 (mean disruption scores 65 and $55 \mathrm{~ms}$, respectively), which in the case of the $b 7$ ran-seq participants cannot have been due to loss of the response sequence. Another five participants ( $b 7$ seq-ran) showed disruption (66 ms) at block 7 despite an absence of relevant explicit knowledge.

\section{Discussion}

The aim of Experiment 3 was to test whether the incidental learning effect, observed in the conjoint sequenced condition (seq-seq) of Experiments 1 and 2, can be firmly attributed to the learning of a sequence of stimulus categories. We hypothesized that if proper task sequence learning, or proper response sequence learning, occurs in the seq-seq condition, the removal of these sequences one at a time will have a different effect on performance than the removal of both at once. However, if the observed learning effect is due to the learning of a sequence of stimulus categories, then no difference between conditions should be expected. The results confirmed the latter hypothesis and suggest that the observed learning effect depends on a sequence of perceptually based stimulus categories rather than a sequence of conceptually based tasks, or even the learning of a motor-based sequence of responses.

We have proposed that disruption in the conjoint sequences conditions of Experiments 1 and 2 was related to the learning of a predictable order of stimulus categories, and the results of Experiment 3 supported this proposition. Obviously, this implies that stimulus category sequence learning depends on the task sequence and response sequence being correlated. As a consequence, the sequence learning effect must disappear when the two sequences differ in length. Under these circumstances the regular integration of tasks and responses is eliminated, such that a predictable sequence of stimulus categories cannot emerge and hence cannot be learned. This hypothesis was tested in Experiment 4.

\section{Experiment 4}

The aim of Experiment 4 was to test whether learning is eliminated when there is no correlation between task sequence and response sequence. We expected that there would be no general disruption in block 7 when uncorrelated sequences were used. Uncorrelated sequences were created by presenting task and response sequences of different lengths.

\section{Method}

\section{Design}

A within-subjects design was administered with the variable block as the only independent variable. 


\section{Materials and procedure}

These were very similar to those in Experiment 2. Task order was sequenced according to a repeating six-element cycle (i.e. colour-task, shape-task, letter-task, shape-task, colour-task, letter-task). Response order was sequenced according to a repeating eight-element cycle, (i.e. $L-R-R-L-R-L-L-R$ ). For the pseudo-random block 7 , the same ordering of tasks and responses was used as in Experiment 2, ran-ran condition.

\section{Results}

Performance across blocks 1-8 was $840,630,601,581$, 572 and 558, 560 and $557 \mathrm{~ms}$, respectively, suggesting a modest training effect across blocks 3-6. However, a one-way ANOVA revealed no significance, with $F(1.8$, $34.7)=2.66, P>0.05$. More importantly, a $t$ test showed no significant difference between block 7 and the average of blocks 6 and 8, $t(19)=0.23, P=0.82$ (two-tailed). Results of the sequence generation test are presented in Table 1. About one-third of the participants correctly generated most or all of the response sequence but little or none of the task sequence.

\section{Discussion}

The aim of Experiment 4 was to further investigate whether the incidental learning effect observed in the seq-seq conditions of the previous experiments was due to the regular integration of tasks and responses into a sequence of stimulus categories. We hypothesized that this effect depends entirely on correlation between task and response sequences. The results of Experiment 4 indeed revealed no indication of conjoint incidental learning when task order and response order were simultaneously sequenced but uncorrelated. As shown in Table 1, explicit response sequence knowledge was reported by only half as many participants in Experiment 4 compared to Experiments 2 (seq-seq condition) and 3, suggesting that it was less salient without the accompanying, and supportive, stimulus category sequence.

\section{General discussion}

The aim of this study was to investigate whether incidental task sequence learning occurs when switching between tasks with univalent stimuli is required in a modified SRT task. Specifically, we asked whether independent task sequence learning can be found when there are no perceptually based task cues. We also asked whether the presence of a perceptually based sequence of stimulus categories plays an important role in incidental task sequence learning. Such an occurrence would be broadly similar to the "unitization" process reported by Goldstone (2000).
Overall, our results provided no evidence for proper task sequence learning. In particular, in the seq-ran condition where only task order, but not response order, was sequenced, there was no indication of incidental task sequence learning. However, there was a reliable learning effect when both task order and response order were sequenced at once. This learning effect emerged to a similar degree when either the task order or the response order was switched to random separately, as well as when the two sequences were switched to random both at once. Moreover, this learning effect materialized only when correlated sequences were used. An evaluation of participants' explicit sequence knowledge revealed no obvious relationship between explicit knowledge and the RT performance measure. Although there was substantial awareness of the response sequence, together with some awareness of the stimulus category sequence (seq-seq correlated condition, Experiments 1-3), no single participant was aware of the task sequence in isolation. One speculative explanation is that, when probed, some participants extrapolated to the task sequence from their knowledge of the stimulus category sequence. Even so, explicit knowledge of the stimulus category sequence was not sufficient to completely account for the disruption effect, and, furthermore, participants who expressed relevant explicit knowledge during questioning, failed to show consistent RT improvement in the earlier part of the experiment.

There are several possibilities why these anomalies might occur. One is that, like the random control participants, some experimental participants correctly generated parts of the sequences purely by guessing. Another is that explicit awareness occurred rather late in the experiment (i.e. most likely just after the introduction of the random block) with some participants engaging in hypothesis testing. A third is that the task requirements were so easy that, after several blocks of practice, any further speed-up of RTs was unlikely - even with some explicit knowledge of the sequence. Incidental learning studies need not assume that performance reflects competence (see Kleinsorge et al. 2003; Shanks and Cameron 2000; Reber and Squire 1998). Most likely, all of these possibilities contributed to the inconsistent effects of explicit sequence knowledge.

To summarize, our results present no firm evidence for incidental task sequence learning per se. Although our experimental testers emphasized the notion of concepts (colour, shape and letter case), once testing got under way participants did not need to think about these concepts any further. With increasing familiarity with the materials, acknowledgement of the different kinds of stimuli and appropriate key-press responses could proceed in an entirely data-driven fashion - without having to name the tasks, or even the stimuli. As response times were generally rather fast, we feel inclined to believe that the tasks were carried out in a more or less automatic way. We would go so far as to suggest that the simple concepts we used actually broke down into percepts 
(redness, blueness, roundedness, etc.) once performance was proceduralized. In other words, as performance changed from controlled to automatic processing, concepts gave way to percepts: hence, where it existed (i.e. seq-seq condition), a sequence of perceptual rather than conceptual components was learned in an incidental way.

Our findings support Heuer et al.'s (2001) interpretation that incidental task sequence learning is very unlikely unless the tasks themselves are "interpreted" in some way. In other words, participants may need to attend and form mental (i.e. conceptual) representations at the level of the tasks (see Jimenez and Mendez 1999; Keele and Jennings 1992; Curran and Keele 1993; Shanks and Channon 2002; Stadler 1995; Berry and Cock 1998, for related discussions). It may well be the case, in our set up at least, that while the response sequence can become salient through attention to the stimulus category sequence (seq-seq condition), the task sequence itself remains hidden, because increasing familiarity with the stimuli-response mappings makes attention to the tasks redundant.

In this respect, it is important to acknowledge that by using univalent instead of bivalent stimuli, selection of the relevant stimulus dimension on any one trial becomes - by definition - unnecessary. It is possible that the performance benefit observed in previous task sequence learning studies is essentially amalgamated with the specific requirements of bivalent stimuli. That is, with bivalent stimuli an additional IF-THEN rule is required for determining which stimulus dimension is relevant for a given task. Incidental task sequence learning, therefore, may be directly related to properties of task-sets involving bivalent stimuli rather than the unique stimulus - task outcome mappings used in the experiments reported here (cf. Rogers and Monsell 1995; Woodward et al. 2003).

Finally, we note that in the real world, rather than being incidental, the learning of a sequence of tasks usually begins under intentional, conscious control with a goal in mind (e.g. publish a scientific paper, paint a room). There is an explicit representation of what is to be achieved even if the elements and the order of elements need to be worked out and perfected. We can think of no situation where a repeated sequence of tasks would require us to make unpredictable choices, or any functional or evolutionary advantage to this. However, if incidental task sequence learning really does occur, and if it is related to the simpler kind of stimulus response-based sequence learning in the laboratory, then a similar mechanism probably applies to both: In the case of the incidental learning of a sequence of real world actions, performance becomes proceduralized through compilation into a chain of stimulus-response associations (Anderson 1990; Pear 2001; Ziessler and Nattkemper 2001). Hence, for the proceduralization of a sequence of tasks to occur, task outcomes must surely always be the same. As a result, a practised sequence of tasks is thereby transformed into a more economic sequence of stimuli and responses.
Acknowledgements We would like to thank Beatrice Hasler, Manette Ruch-Monachon and Yves Steiner for running the experiments and Pierre Perruchet for his useful comments on an earlier draft of the manuscript.

\section{References}

Ahissar M, Hochstein S (1995) How early is early vision? Evidence from perceptual learning. In: Papathomas T, Chubb C, Gorea A, Kowles E (eds) Early vision and beyond. MIT Press, Cambridge, pp 199-206)

Anderson JR (1990) Cognitive psychology and its implications. W.H. Freeman and Company, New York

Bapi RS, Doya K, Harver AM (2000) Evidence for effector independent and dependent representations and their differential time course of acquisition during motor sequence learning. Exp Brain Res 132:149-162

Berry DC, Cock J (1998) Implicit learning of invariant features? In: Stadler MA, Frensch PA (eds) Handbook of implicit learning. Sage Publications, Thousand Oaks, pp 135-160

Cock J, Berry DC, Buchner A (2002) Negative priming and sequence learning. Eur J Cognit Psychol 14:27-48

Cohen A, Ivry RI, Keele SW (1990) Attention and structure in sequence learning. J Exp Psychol Learn Mem Cogn 16:17-30

Curran T, Keele SW (1993) Attentional and nonattentional forms of sequence learning. J Exp Psychol Learn Mem Cogn 19:189-202

Frensch PA, Miner CS (1995) Effects of presentation of rate and of individual differences in short-term memory capacity on an indirect measure of serial learning. Mem Cogn 22:95-110

Goldstone RL (2000) Unitization during category learning. J Exp Psychol Hum Percept Perform 26:86-112

Goschke T (1998) Implicit learning of perceptual and motor responses. In: Stadler MA, Frensch PA (eds) Handbook of implicit learning. Sage Publications, Thousand Oaks, pp 401-444

Gotler A, Meiran N, Tzelgov J (2003) Nonintentional task set activation: evidence from implicit task sequence learning. Psychon Bull Rev 10:890-896

Heuer H, Schmidtke V, Kleinsorge T (2001) Implicit learning of sequences of tasks. J Exp Psychol Learn Mem Cogn 27:967-983

Hoffmann J, Koch I (1997) Stimulus-response compatibility and sequential learning in the serial reaction time task. Psychol Res 60:87-97

Hoffmann J, Sebald A (1996) Reiz- und Reaktionsmuster in seriellen Wahlreaktionen. Z Exp Psychol 43:40-68

Humphreys GW, Quinlan PT, Riddoch MJ (1989) Grouping processes in visual search: effects with single and combined-feature targets. J Exp Psychol Gen 118:258-279

Jimenez L, Mendez C (1999) Which attention is needed for implicit sequence learning? J Exp Psychol Learn Mem Cogn 25:236-259

Keele SW, Jennings PJ (1992) Attention in the representation of sequence: experiment and theory. Hum Mov Sci 11:125-138

Kleinsorge T, Schmidtke V, Gajewski PD, Heuer H (2003) The futility of explicit knowledge of a sequence of tasks. Eur $\mathbf{J}$ Cognit Psychol 15:455-469

Koch I (2001) Automatic and intentional activation of task sets. J Exp Psychol Learn Mem Cogn 27:1474-1486

Koch I, Hoffmann J (2000) The role of stimulus-based and response-based spatial information in sequence learning. J Exp Psychol Learn Mem Cogn 26:863-882

Krueger LE (1984) The category effect in visual search depends on physical rather than conceptual differences. Percept Psychophys 35:558-564

Lashley KS (1951) The problem of serial order in behavior. In: Jeffress LA (ed) Cerebral mechanisms in behaviour. Wiley, New York

Marsolek CJ, Field JE (1999) Perceptual-motor sequence learning of general regularities and specific sequences. J Exp Psychol Hum Percept Perform 25:815-836

Mayr U (1996) Spatial attention and implicit sequence learning. Evidence for independent learning of spatial and nonspatial sequences. J Exp Psychol Learn Mem Cogn 22:350-364 
Nissen MJ, Bullemer P (1987) Attentional requirements of learning: evidence from performance measures. Cognit Psychol 19:1-32

Pear JJ (2001) The science of learning. Psychology Press, Philadelphia

Perruchet P, Amorim M-A (1992) Conscious knowledge and changes in performance in sequence learning: evidence against dissociation. J Exp Psychol Learn Mem Cogn 18:785-800

Reber PJ, Squire LR (1998) Encapsulation of implicit and explicit memory in sequence learning. J Cognit Neurosci 10:248-263

Rogers RD, Monsell S (1995) The cost of a predictable switch between simple cognitive tasks. J Exp Psychol Gen 124:207-231

Schmidtke V, Heuer H (1997) Task integration as a factor in secondary-task effects on sequence learning. Psychol Res 60:53-71

Shanks DR, Cameron A (2000) The effect of mental practice on performance in a sequential reaction time task. J Motor Behav 32:305-313

Shanks DR, Channon S (2002) Effects of a secondary task on "implicit" sequence learning: learning or performance? Psychol Res 66:99-109

Shanks DR, Johnstone T (1998) Implicit knowledge in sequential learning tasks. In: Stadler MA, Frensch PA (eds) Handbook of implicit learning. Sage Publications, Thousand Oaks, pp 533-572

Shanks DR, Johnstone T (1999) Evaluating the relationship between explicit and implicit knowledge in a sequential reaction time task. J Exp Psychol Learn Mem Cogn 25:1435-1451
Shin JC, Ivry RB (2002) Concurrent learning of temporal and spatial sequences. J Exp Psychol Learn Mem Cogn 28:445-457

Stadler MA (1995) Role of attention in sequence learning. J Exp Psychol Learn Mem Cogn 21:674-685

Willingham DB (1998) A neuropsychological theory of motor sequence learning. Psychol Rev 105:558-584

Willingham DB, Nissen MJ, Bullemer P (1989) On the development of procedural knowledge. J Exp Psychol Learn Mem Cogn 15:1047-1060

Willingham DB, Greeley T, Bardone AM (1993) Dissociation in a serial response time task using a recognition measure. Comment on Perruchet and Amorim. J Exp Psychol Learn Mem Cogn 19:1424-1430

Wolfe JM, Friedman-Hill SR, Stewart MI, O'Connell KM (1992) The role of categorization in visual search for orientation. J Exp Psychol Hum Percept Perform 18:34-49

Woodward T, Meier B, Tipper C, Graf P (2003) Bivalency is costly: bivalent stimuli elicit cautious responding. Exp Psychol 50:233238

Ziessler M (1994) The impact of motor responses on serial pattern learning. Psychol Res 57:30-41

Ziessler M, Nattkemper D (2001) Learning of event sequences is based on response-effect learning: further evidence from a serial reaction time task. J Exp Psychol Learn Mem Cogn $27: 595-613$ 